\title{
Analysis on the Characteristics of Wind Power Output in Hainan Power Grid*
}

\author{
Jianfeng Wang, Dongmei Zhao \\ College of Electrical and Electronic Engineering, North China Electric Power University, Beijing, China \\ Email:wjfwjfcool@126.com \\ Received March, 2013
}

\begin{abstract}
It is of great importance to study the characteristics of wind power output for the healthy and secure \& stable of power grid. Based on the actual operating data, the probability distribution of the power fluctuations of the wind farm in Hainan and the variation of wind power annual, seasonal, daily active output is analyzed. The study showed that Hainan Province has obvious seasonal variation of wind power output characteristics, higher levels of output of the year generally in winter or summer, spring and autumn to contribute small. The average wind power output will contribute to "low day and high night”, with certain peaking capacity. Shorter time scales, changes in the wind power to smaller amount, not to bring too much impact on system operation, while a long time fluctuations affect the scheduling and running on the grid.
\end{abstract}

Keywords: Wind Power; Power Fluctuation; Probability Distribution

\section{Introduction}

With the growing energy and environmental problems, the development of new energy has been a concern around the globe. China has a vast coastline of wind energy resources are widely distributed and relatively rich areas are mainly concentrated in the southeast coast and nearby islands and the northern. In addition, inshore and offshore wind energy resources are very rich [1].

The new energy is one of the pillar industries in recent years to focus on supporting the development of Hainan, Hainan wind power has been rapid development. As of the end of June this year, Hainan new energy installed capacity of 249.5 MW wind power project to dominate. Last year, Hainan wind power generation of 270 million kWh.

Because of intermittent, randomness and volatility characteristics of wind, with the rising proportion of wind power installed capacity proportion in the system, the impact on security, stability and economic operation of the power system will cannot be ignored[2,3]. Wind power output change by a variety of geographical and climatic factors. Usually only by the statistics and analysis of a large number of actual data, can we get the variation of the wind power in particular areas. Therefore, based on the actual operating data of Hainan several

${ }^{*}$ The National High Technology Research and Development of China 863 Program (2012AA050201). wind farms from 2011 to 2012, variation of wind power annual, seasonal, daily active output is analyzed. Further with the probability distribution method, the fluctuations of wind power are quantitative analyzed, as well as the impact on Hainan power grid.

\section{Overview of Wind Power in HAINAN}

Hainan Island is located in northern margin of tropical, with tropical monsoon maritime climate. Winter prevailing northeast monsoon and prevailing southwest monsoon in the summer, sometimes blowing southeast monsoon and many tropical cyclones occurs. The wind energy resources which can be developed and used are mainly distributed in the coastal areas, offshore areas and some inland mountainous area.

Off the coast of Hainan is rich in wind energy resources, and the average wind speed in most parts is between $4.3 \mathrm{~m} / \mathrm{s}$ to $5.2 \mathrm{~m} / \mathrm{s}$. As of the end of 2012, the Hainan power grid has been put into operation in wind farms in six, the total installed capacity of 303MW, accounting for about $7.23 \%$ of the installed capacity of Hainan province. The six grid wind farms are Wenchang wind farm, E'man wind farm, Gancheng wind farm, Sigeng wind farm, Gaopai wind farm and Dongfang wind farm, as shown in Figure 1. The capacity of Dongfang wind farm is very small, and Gaopai just put into operation in the end of the year, in this paper, the first four wind farm will be the research focus. 


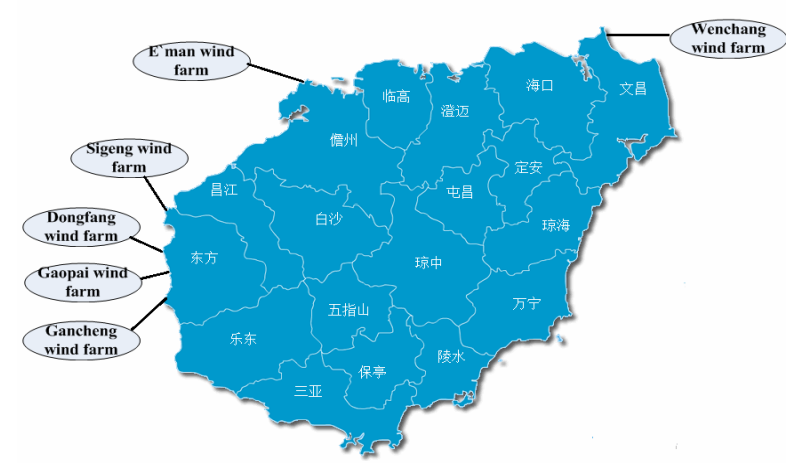

Figure 1. Diagram of wind power in Hainan.

\section{Wind Power Output Fluctuation Analyses}

\subsection{Output Level under Different Years}

Over a period of time, the wind power output level is constantly in random fluctuations, it is difficult to accurately predict, therefore, need to select an indicator to study the laws of statistics. Wind power annual utilization hours [4], also known as Equivalent full load power generation hours, Refers to the ratio of the actual generating capacity of wind power equipment in the year with annual generating capacity of the power generation equipment running at rated power. Statistics Hainan wind farm operating data in the past two years, the annual utilization hours is about $1433 \sim 2340 \mathrm{~h}$, the average value of 2000h. Annual utilization hours of wind power affected by multiple factors: 1) the impact of meteorological factors such as wind conditions, climate, natural disasters, etc; 2) wind turbine failure rate, reliable operation time of the unit; 3 ) the transmission, substation capacity constraints of wind farm area; 4)the electric field losses, transformer, line losses, and other auxiliary power consumption.

Wind power annual utilization hours can evaluate the level of efficiency in the use of the wind farm, the level of annual utilization hours reflect the relative size of the level of wind speed from the other side in different years. Figure 2 shows, the average wind speed of each region in 2012 is basically lower than 2011 .

\subsection{Wind Power in Different Seasons and Months}

The wind speed of an area is largely influenced by the local climate, wind energy resources of monsoon climate region shows apparent regularity in the long-term within the one-year cycle. Active power and capacity factor [5] was chosen as indicators in the Statistics of the output data of the Hainan four wind farms in 2011 and 2012. The results are shown in Figure 3.

In 2011 and 2012, the maximum output occurs in January and June, with the value 83.3MW and 56.5MW respectively; Minimum output occurs in August and September, 14.8 MW and 22.7 MW respectively. The seasonal maximum peak-to-valley was $68.5 \mathrm{MW}$ and 33.8 MW, accounting for $37.18 \%$ and $18.31 \%$ of the wind turbine total installed capacity.

From the wind power output curves in these two years we can find, generally the largest wind power output appear in the November, December, January, Under the influence of the winter monsoon, Hainan have a greater average wind speed; In the summer, around June, will also appear larger wind, which is related to the impact of the monsoon, and tropical cyclones; spring and autumn wind is small generally.

Figure 4 shows annual capacity factor curve in the Wenchang, E'man, Gancheng and Sigeng wind farm in 2011. The curve shows, in the winter, the output of all the wind farms have reached the peak level of the year;

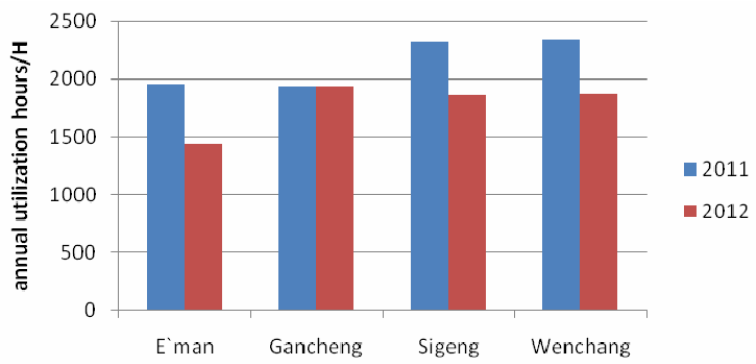

Figure 2. Annual utilization hours in different wind farms.

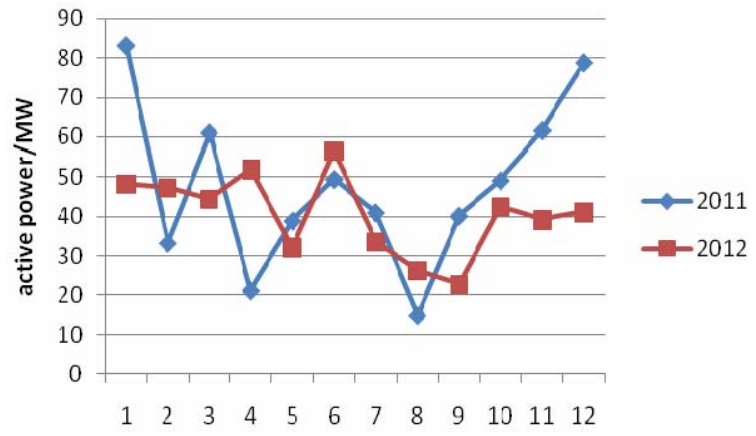

Figure 3. The total power of the four wind farms in each month.

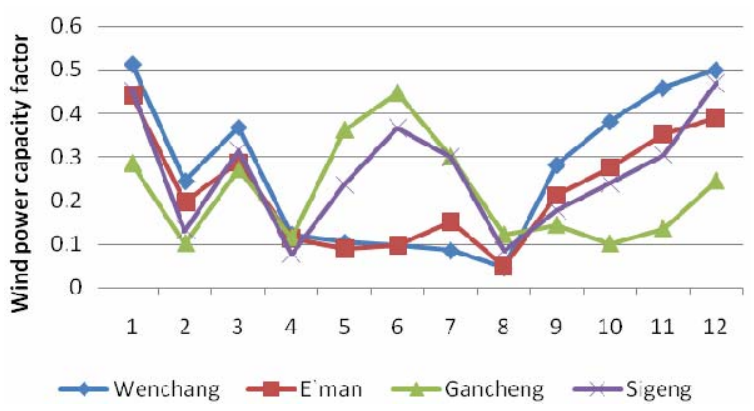

Figure 4. Annual capacity factor curve in the four wind farms in 2011. 
in spring and autumn, wind power output is lower; in the summer, different wind farm have different variation.

The geographic distance between Gancheng wind farm and Sigeng wind farm is very near, at the west of Hainan Island, has a similar law for the wind power output fluctuations. The curves present bimodal characteristics, winter and summer wind power output is high, while spring and autumn to contribute significantly lower, which exhibit significant characteristics of the monsoon. On the other hand, Wenchang and E'man, at the north of Hainan, have a different variation rule. In winter, wind speed and wind power capacity factor are much higher than other periods, in spring, summer and autumn wind is low, and relatively little change, Curve shows unimodal characteristics.

\subsection{Wind Power Output Changes in One Day}

For power systems, wind power is an uncontrollable power source, the increase in power output of wind power, means that the system equivalents loads is relatively small, and further affect daily open formulation and adjustment of the shutdown plan of the power system. Therefore, it is necessary to study wind power output variation in 24 hours.

With the active output data of wind farms in Hainan in 2012 as the foundation, the variation of the capacity factor of each power plant in one day is calculated, as shown in Figure 5.

It can be found in a wide range of areas in Hainan, the capacity factor of wind farms in 24 hours with the same regularity. Wind power output level is low in the night and the morning, and little change; Afternoon, the wind power output level is increasing, and the peak generally appear in the 14:00 to 17:00. We can find that wind power output of Hainan, which is unlike in inland areas of significant anti-peaking[6] characteristics presents the characteristics of "low day and high night" and has certain support and added effect to peak load regulation in power system, on the other hand, it is also conducive to the elimination of the grid for wind power. In the mentioned four wind farm, E'man, Gancheng Sigeng wind power output shows obvious fluctuations trend in one day, the peak output level can reach twice of the night, while power output is more tend to steady in Wenchang, only a slight increase in the afternoon.

In order to study how the wind farm daily output curves changes under different seasons, E'man wind farm is made as an example for analysis on daily output level in each season, as shown in Figure 6.

E'man wind farms wind power output has almost the same change trend in different season, showed a single peak characteristic, which usually appear at 14:00 to $17: 00$, the affection of the seasonal variation on wind power output is mainly reflected in the size of the spe- cific values. Clearly, wind power capacity factor is basic above 0.4 in winter, Indicating that higher utilization efficiency in winter; While in spring, for a very long time, the wind power capacity factor is less than 0.2 .

\section{Probability Distribution of Wind Power}

\subsection{Probability Distribution of Wind Speed}

The distribution characteristics of wind speed generally shows positive skewness, Weibull distribution [7, 8] is generally considered as a suitable probability density function for the wind speed statistical description. The Weibull distribution is a single peak distribution function cluster, which has two parameters. Its probability density function can be expressed as:

$$
p(x)=\frac{k}{c}\left(\frac{x}{c}\right)^{k-1} \exp \left[-\left(\frac{x}{c}\right)^{k}\right]
$$

where, $\mathrm{k}$ is called the shape parameter, c called the scale parameter.

There are a variety of methods to estimate parameters of the Weibull distribution, which is chosen depending on the wind speed statistics. Three methods are commonly used [9]: Least squares method, mean and variance estimation method, minimum error approximation method.

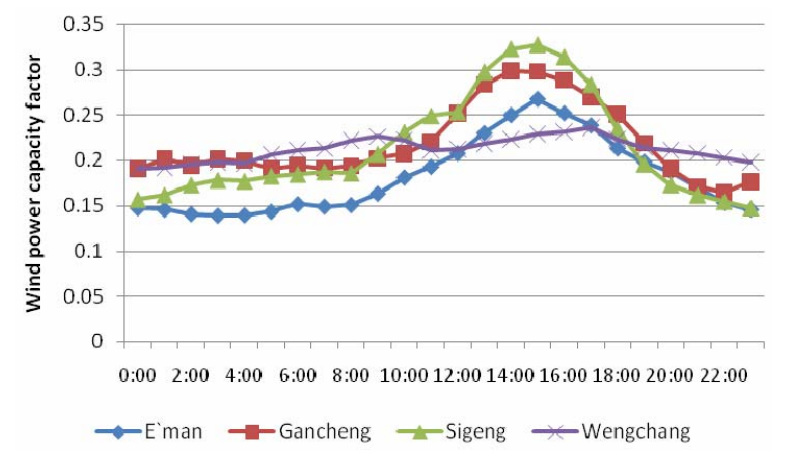

Figure 5. Wind power capacity factor in E'man, Gancheng Sigeng and Wenchang wind farm in $24 \mathrm{~h}$.

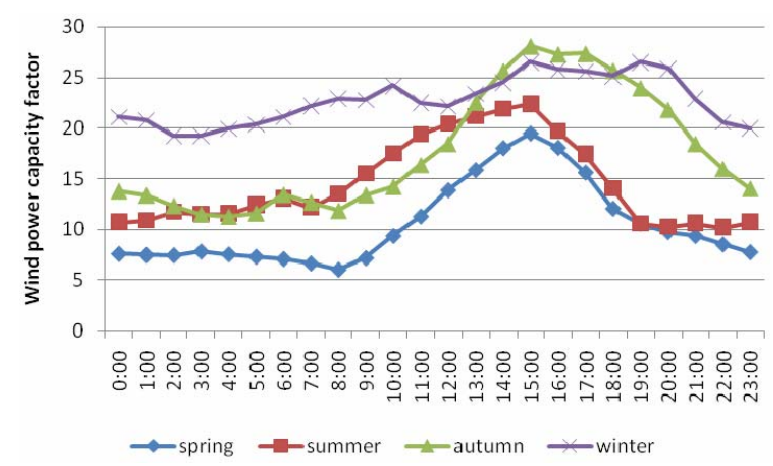

Figure 6. Wind power capacity factor in E'man in each season. 
According to the statistics of wind speed data in 2012, combined with the second method, the calculated wind speed distribution parameters are as follows in Table 1:

\subsection{Probability Distribution of Wind Power Fluctuations}

At present, the quantitative analysis of the characteristics of wind power fluctuations is few, the probability distribution of the field is also not very mature. The normal distribution can be used to describe the distribution of the first-order differential sequence of wind power $[10,11]$ proposed an improved t-distribution to describe the minute level power fluctuation. The probability distribution of the power differential sequence has an important role to researcher like wind power forecast, wind farms equivalents modeling and so on, so following this detailed study:

In order to quantify the power fluctuations of the wind power, this article refers to two numerical feature amounts to describe the first-order differential sequence of wind power. Assuming that $P$ as the wind power of a wind farm at a certain moment, where $P$ is an n-dimensional vector, $n$ is number of the wind farm units, the average of wind power is $\bar{P}$, and using the standard deviation of wind power output $S$ as quantitative indicators to describe the amplitude of the wind power fluctuation.

$$
S=\sqrt{\frac{1}{n} \sum_{i=1}^{n}\left(P_{i}-\bar{P}\right)^{2}}
$$

Assuming that $T$ is to describe the probability of occurrence of the first-order differential sequence of wind power at different amplitude range [12]. The following formula is:

$$
T=N_{\Delta p} / N
$$

where: $N_{\Delta p}$ is the number of occurrences of a certain range of first-order differential sequence, $N$ is the total number of wind power differential sequence.

Statistical analysis is to used on Gancheng wind farm actual operating data of one day in2011, installed capacity of $49.5 \mathrm{MW}$. The distribution of the amplitude of power fluctuation at different time scales (10 s, 1 min, 15 min, 1 h) is shown in Figure 7.

Table 1. Wind speed probability distribution parameters in four wind farms.

\begin{tabular}{ccccc}
\hline Wind farm & $\begin{array}{c}\text { Reference } \\
\text { height/m }\end{array}$ & $\begin{array}{c}\text { average wind } \\
\text { speed/(m/s-1) }\end{array}$ & c & $\mathrm{k}$ \\
\hline E’man & 65 & 6.24 & 6.12 & 2.01 \\
Gancheng & 65 & 6.74 & 5.99 & 1.63 \\
Sigeng & 65 & 6.12 & 6.09 & 2.01 \\
Wenchang & 65 & 6.38 & 6.14 & 1.97 \\
\hline
\end{tabular}

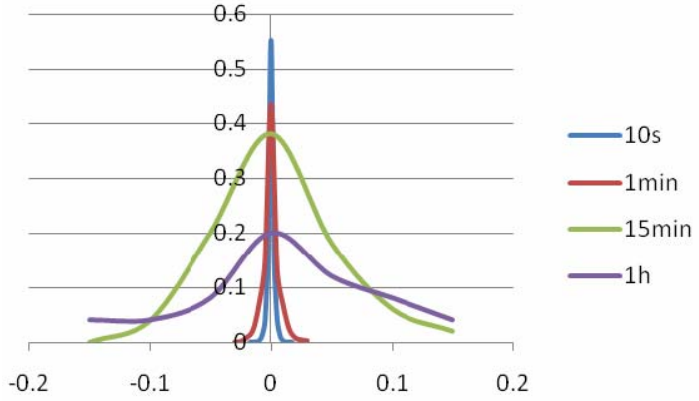

Figure 7. Distribution of the amplitude of power fluctuation at different time scales.

Table 2. Wind power maximum, and standard of the first-order differential sequence.

\begin{tabular}{ccccc}
\hline Time scale & $10 \mathrm{~s}$ & $1 \mathrm{~min}$ & $15 \mathrm{~min}$ & $1 \mathrm{~h}$ \\
\hline $\begin{array}{c}\text { Power fluctuations } \\
\text { maximum (MW) }\end{array}$ & 0.871 & 1.875 & 6.698 & 6.564 \\
$\begin{array}{c}\text { Standard deviation } \\
\text { (MW) }\end{array}$ & 0.145655 & 0.400795 & 1.872348 & 3.588958 \\
\hline
\end{tabular}

At $1 \mathrm{~min}, 15 \mathrm{~min}$ scale, the probability of the distribution in $\pm 0.01 p u$ is $85.25 \%$ and $76.0 \%$, respectively; at $10 \mathrm{~s}-15 \mathrm{~min}$ scale, the probability of the fluctuations of wind power in $\pm 0.01 \mathrm{pu}$ is almost $100 \%$.

With increased sampling time scale, the magnitude of the wind power fluctuations increases, the distribution area of power fluctuations will be more widely. Table 2 from the perspective of the active wind power fluctuation maximum value and the standard deviation, indicating that wind power fluctuations grows with the larger time scale.

In the very small time scale, power fluctuations is a smaller amount, does not bring too much impact on the system operation; However, when the size of the gridconnected wind power increasing and wind power penetration is high, which will cannot be ignored. The short-term fluctuations affect system infrequency modulation, and long-term fluctuations have effect on dispatching and operation of power system.

\section{Conclusions}

Based on Hainan wind farm actual operating data, from the two aspects of the time scale and the probability density distribution, in this paper, the wind power output in different situations are compared and studied, the conclusion is as follows:

1) In Hainan, wind farms are mainly located on the west coast and Wenchang, belongs to the offshore wind power; the annual utilization hours is about $1433 \sim 2340 \mathrm{~h}$, the average value of $2000 \mathrm{~h}$, wind energy resources are relatively abundant.

2) The output of wind power has obvious seasonal 
variation characteristics: in winter, wind power output is at the peak, and there is a clear correlation; the output of spring and fall of each wind farm is low;

3) Unlike inland wind power's "high day and low night" feature, each wind farm, in every season, the wind power output is basically the same, peak generally appear in the afternoon from 2 o'clock to five o'clock, showed a single peak characteristics conducive to the system peaking wind power consumption;

4) Shorter time scales, changes in the wind power to smaller amount, not to bring too much impact on system operation, long time fluctuations affect the scheduling and running on the grid, the need for further research.

\section{REFERENCES}

[1] J. F. Li, “China Wind Power Outlook 2012,” Beijing: China Environmental Science Press, 2012.

[2] W. Y. Li, B. H. Zhang and Bagen, "Reliability Impacts of Large Scale Utilization of Wind Energy on Electric Power Systems," Proceedings of the CSEE, Vol. 28, No. 1, 2008, pp.100-105.

[3] J. Guo, "Study on Impact of Grid-connected Wind Turbine \& Wind Farm on Power System," The New Energy Power Control Technology, Vol. 32, No. 1, 2010, pp. 47-50.

[4] Z. S. Lin and L. Q. Luo, "Analysis of Wind Power Output Characteristics in Fujian Andits Impact on Power Grid," Electric Power Construction, Vol. 32, No. 12, 2011, pp. 18-23.

[5] D. Y. Yu, X. S. Han, J. Liang and S. G. Song, "Study on the Profiling of China's Regional Wind Power Fluctuation Using GEOS-5 Data Assimilation System of Na- tional Aeronautics and Space Administration of America,” Automation of Electric Power System, Vol. 35, No. 5, 2011, pp. 77-81.

[6] L. D. Yi, M. Y. Zhu, L. Wei, N. Jiang and G. L. Yu, “A Computing Method for Peak Load Regulation Ability of Northwest China Power Grid Connected With Large-Scale Wind Farms,” Power System Technology, Vol. 34, No. 2, 2010, pp. 129-132.

[7] G. Yang, Y. X. Du and M. Chen, "The Calculation of Energy Provided by Wind Turbine Based on Its Power Characteristic and the Wind Frequency Weibull Distribution,” Journal of Electric Power, Vol. 23, No. 4, 2008, pp. 276-278.

[8] M. Ding, W. Wu, H. B. Wu, M. Q. Mao and Y. Y. Zhang, "Research on Forecasting of Probabilistic Distribution Parameters of Wind Speed and Its Application," Power System Technology, Vol. 32, No. 14, 2008, pp. 10-14.

[9] M. Ding, Y. C. Wu and L. J. Zhang, "Study on the Algorithm to the Probabilistic Distribution Parameters of Wind Speed in Wind Farms," Proceedings of the CSEE, Vol. 25, No. 10, 2005, pp. 107-110.

[10] L. Xiang, K. Chang, X. Y. Li and F. Xue, "Probe into Temporal-spatial Characteristics of Wind Power Output in Ningxia,” Shaanxi Electric Power, 2011, pp. 43-49.

[11] W. X. Lin, J. Y. Wen, X. M. Ai, S. J. Cheng and W. J. Lee, "Probability Density Function of Wind Power Variations," Proceedings of the CSEE, Vol. 32, No. 1, 2012, pp. 38-46.

[12] Y. Cui, G. Mu, Y. Liu and G. H. Yan, "Spatiotemporal Distribution Characteristic of Wind Power Fluctuation," Power System Technology, Vol. 35, No. 2, 2011, pp. 110-114. 